\title{
Experimental and numerical analysis of an oil- flooded air screw expander
}

\author{
Lukas Richter ${ }^{1 *}$, Zdenka Rendlova ${ }^{1}$, Stanislav Planicka ${ }^{1}$, Jan Seeger $^{2}$, Marco Taschek ${ }^{2}$, \\ Michal Volf ${ }^{1}$, Martin Pelikan ${ }^{1}$ and Vit Linhart ${ }^{3}$ \\ ${ }^{1}$ University of West Bohemia in Pilsen, Univerzitni 22, 30614 Pilsen, Czech Republic. \\ ${ }^{2}$ OTH Amberg-Weiden, Kaiser-Wilhelm-Ring 23, 92224 Amberg, Germany. \\ ${ }^{3}$ ATMOS vyvoj s.r.o., Plzenska 149, 33003 Chrast, Czech Republic.
}

\begin{abstract}
This article describes the results of a project whose task is to research and develop a screw expander through the inversion of a screw compressor. The article summarizes aspects of the construction of an experimental device that works with an oil-flooded air screw expander. The expander was subjected to experimental and numerical analysis, the results of which are presented in the article. Numerical analysis to examine the expansion process is performed both on the basis of the analytical geometric description of the working chamber of the expander and on the basis of the geometry obtained from a 3D scan of a real machine. The results of experimental and numerical analysis will be used to integrate an oil-flooded screw expander into an energy unit for the use of low-potential heat, for example in ORC systems.
\end{abstract}

\section{Introduction}

A screw expander is based on the inversion of a screw compressor and under certain conditions may be a suitable alternative to a turbine as a thermal cycle expansion device for the transformation of thermal energy into mechanical energy. These conditions are basically defined by the parameters of the heat source. A screw expander can be used in particular when the heat source is limited, which is typical, for example, with solar or geothermal energy and for the use of waste heat. The classical steam Rankine cycle is not suitable for the use of low-potential heat, and therefore working substances with a lower boiling point than water are usually used. If such a working substance is used, a screw expander is usually an integral part of the Organic Rankine Cycle (ORC), the binary cycle or the Kalina cycle. In the case of low-potential heat applications, a liquid phase may appear during the expansion of the low-boiling working fluid, causing erosion of the turbine blades. In such a case, a screw expander capable of working without damage should be used, even with a relatively large proportion of liquid phase. For example, the trilateral flash cycle (TFC), for which wet expansion is typical, may be suitable for the use of flow-limited heat sources.

In the case of low-potential heat applications, a screw expander is characterized by the relatively low velocity of the working medium, low speed and a lower flow rate of the

\footnotetext{
* Corresponding author: richterl@kke.zcu.cz
} 
working medium, unlike microturbines. Microturbines for the use of low-potential heat tend to be high-speed (tens of thousands of RPM) and their phasing into the electrical grid with a frequency of 50 or $60 \mathrm{~Hz}$ is more demanding. Nowadays, it is possible to produce a screw expander with a maximum rotor diameter between $800-900 \mathrm{~mm}$, which corresponds to a maximum output power of approximately $5 \mathrm{MW}$. However, for rotor diameters greater than $350 \mathrm{~mm}$, production costs rise steeply, and therefore the ideal power output range for a screw expander is limited to approximately $50 \mathrm{~kW}-500 \mathrm{~kW}$. The higher the accuracy of the screw expander production, the higher the efficiency of the expander can be achieved. On modern milling and grinding machines, high precision screw rotor production can be achieved with a tolerance ranging between 3 - $5 \mu \mathrm{m}[1]$.

The aim of our research, the outputs of which are described in the article, is to perform an analysis of the expansion process in an oil-flooded air screw expander using experimental and numerical approaches. The results are used to implement a screw expander into an energy unit for the use of low-potential waste heat. In addition, the mathematical model can be used to modify the profiles of screw bodies to optimize the expansion process.

\section{Working principle of oil-flooded screw expander}

Screw expanders can be made in many variants depending on the number of rotors, different profiles of the screw bodies or on the number of rotor teeth. The different configurations usually depend on the pressure conditions, lubrication or expander speed. The expander we analysed has two rotors (twin screw expander). The male rotor connected to the shaft has 4 lobes and the female rotor has 5 lobes. The rotors together with the expander casing form the working chamber, the volume of which first increases through isobaric filling up to the value defined by the geometry of the inlet port. As the rotation continues, the chamber closes and separates from the inlet port. However, the volume of the working chamber continues to grow, and therefore there is isentropic expansion and a decrease in the pressure of the working fluid. At the moment when the working chamber is exposed to the low-pressure port, the fluid is gradually discharged isobarically. Continued rotation leads to a reduction of the contact line between the teeth to the level of zero volume of the working chamber, when the fluid is completely discharged. The whole process of gradual filling, expansion and discharge is thus completed after two revolutions of the main rotor $\left(720^{\circ}\right)$. This working principle is shown in Fig. 1 [2].

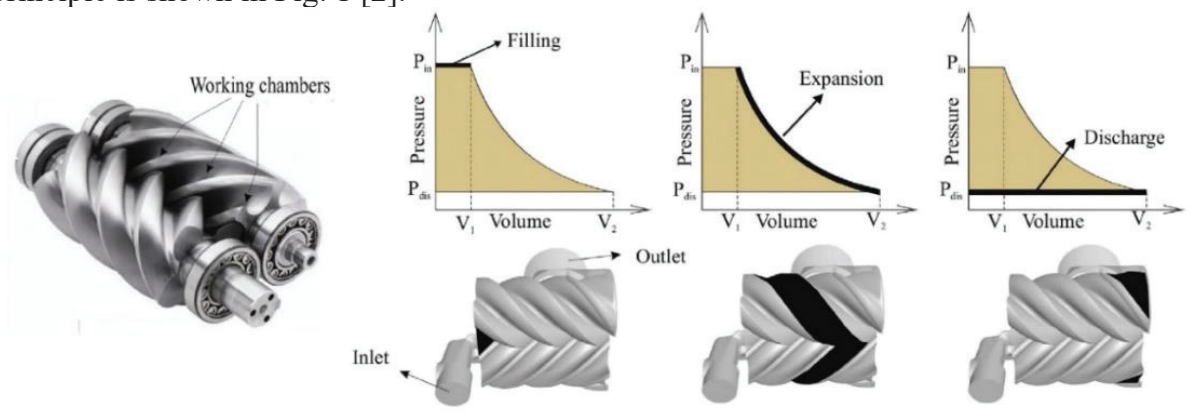

Fig. 1. Working principle of a screw expander [2].

The volume of the working chamber should be as large as possible, thus maximizing the discharge of fluid per revolution. The profiling of the rotors must be designed so that an oil sealing line is formed in each contact position both between the rotors and between the rotors and the casing, because leakage of the working fluid from the working chamber adversely 
affects the efficiency of the screw expander. On the other hand, the contact forces must be low so that the rotors do not deform due to friction losses and the local temperature increases.

The operation of an oil-flooded screw expander depends on a relatively large flow of lubricating oil, which is injected into the working chamber at predetermined locations. The oil is used to lubricate the rotor motion and thus to reduce friction losses, as well as to seal gaps and for cooling when the machine is operated in compressor mode. Oil-flooded machines have a simple mechanical design that requires an external shaft seal, but no internal seal. A thin oil film is formed between the rotors, which seals the inter-rotor clearance, resulting in low internal leakage. However, the viscosity of the oil limits the maximum speed at the top of the rotor. Its value can vary along the rotors depending on the temperature, but it must always comply with the specified limits. Typical values are about $50 \mathrm{~mm}^{2} / \mathrm{s}$, and this value decreases with increasing temperature [1].

\section{Construction of test bench with oil-flooded screw expander}

At first glance, it seems that the oil-lubricated screw expander is a simple inversion of a conventional oil-flooded screw compressor. A closer look shows that this is not the case at all. The main differences to be examined relate to the transmission of power between the rotors, the replacement of the self-circulating oil system by forced circulation, the regulation and control of the rotational speed, the separation of the oil from the expanded air under low pressure and high volume flow conditions, and subcooling of the working fluid during expansion (unlike the compression process, where heat is generated). Considering that in the case of expansion, lubricating oil does not act as a cooling medium (as opposed to compression), its quantity and injection method seem to be important for the generated power.

The test bench can be divided into 3 basic parts - a) compressor unit to create compressed air, b) screw expander and its oil management, c) shaft measurement and electrodynamic brake.

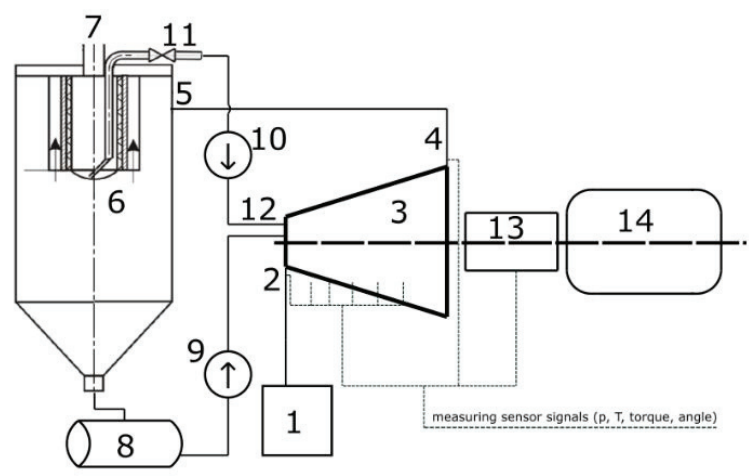

a)

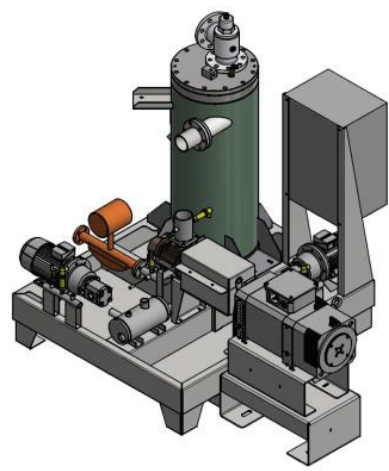

b)

Fig. 2. a) simplified 1-D schematic of test bench, b) 3D CAD model of test bench.

The function can be described as follows according to Fig. 2(a). The compressor (1) generates compressed air which enters the expander through the high-pressure port (2). In the oilflooded expander (3), air is mixed with lubricating oil, which enters the expander through the oil ports (12). Air expands in the expander and the entire mixture of air and oil then exits the expander through the low-pressure port (4). The amount of oil in the expanded mixture is around $5 \mathrm{~kg}$ per cubic metre of air. The expanded mixture then enters tangentially (5) into the oil separator (6). The oil separator vessel is designed as a cyclone separator in 
combination with a fine filter for separating residual oil. Based on the centrifugal principle of the cyclone, most of the oil is trapped at the bottom of the vessel. However, the air separated by the cyclone still contains a residual amount of oil $\left(1-5 \mathrm{~g} / \mathrm{m}^{3}\right)$, and therefore this mixture further passes through a filter membrane, where the residual oil is separated. Purified air with an oil content of less than $3 \mathrm{mg} / \mathrm{m}^{3}$ then emerges from the vessel through the upper lid (7). Because the oil separation takes place under conditions of low pressure and relatively large volume flow, a separation vessel of relatively large dimensions was chosen (diameter $419 \mathrm{~mm}$ and height $1030 \mathrm{~mm}$ ). From the bottom of the separation vessel, the oil is then drained into the oil collecting tank (8) and from there pumped (9) back into the screw block. Residual oil trapped by the fine filter is also pumped (10) back to the screw block by a scavenge line (11). The test bench is further equipped with a torque measuring shaft (13) and an electrodynamic brake (14) for controlling the speed and torque of the expander.

\section{Screw expander analysis}

\subsection{Theoretical assumptions}

The screw expander is essentially a volumetric device, in other words also a positive displacement device. From this point of view, its function can be described by means of volumetric efficiency. Volumetric efficiency is defined as the ratio of actual and theoretical volume flow, and thus can also be expressed in terms of the ratio of actual and theoretical mass flow. However, in the case of a screw expander, the actual flow may be higher than the theoretical flow, which would mean a volumetric efficiency higher than 1. For this reason, the ratio of the actual and theoretical flow is referred to as the Filling Factor (FF) [2]:

$$
F F=\frac{\dot{m}_{\text {real }, \text { fill }} \cdot v\left(T_{\text {fill }}, p_{\text {fill }}\right)}{n_{m r} \cdot z_{m r} \cdot V_{\text {fill }}}
$$

Where $\dot{m}_{\text {real,fill }}$ is actual mass flow of the working fluid during filling $\left[\mathrm{kg} \cdot \mathrm{s}^{-1}\right]$,

$v\left(\mathrm{~T}_{\text {fill }}, p_{\text {fill }}\right)$ is specific volume as a function of filling temperature and pressure $\left[\mathrm{m}^{3} \cdot \mathrm{kg}^{-1}\right]$,

$n_{m r}$ is rotational speed of the male rotor $\left[1 \cdot s^{-1}\right]$,

$z_{m r}$ is number of teeth (male rotor),

$V_{\text {fill }}$ is the filling volume $\left[\mathrm{m}^{3}\right]$.

During the operation of the expander, two main phenomena affecting the FF value occur. These are throttling losses and internal leaks. As the rotors rotate, the entire area of the inlet port is only open for a short time, and therefore throttling losses occur, which reduce the actual flow and thus the FF value. Internal leakage of working fluid occurs between the individual working chambers in the direction from the high-pressure port to the low-pressure port. As a result, additional mass is required at the inlet to the expander, which increases the $\mathrm{FF}$ value (possibly above 1). The value of FF varies depending on the pressure ratio and especially on the rotational speed. Higher admission pressure results in more intense internal leaks and thus a higher FF value, however, this phenomenon is also compensated by increasing pressure loss at the inlet port, which reduces the mass flow. The rotation speed has an even greater effect on the FF value. Higher speed causes an increase in throttling losses and thus a decrease in the Filling Factor, because the relative effect of internal leaks is smaller and the pressure loss at the inlet port increases. It is evident that the best results are obtained when the value of the Filling Factor is close to one [2,3].

The significance of internal leaks decreases more or less linearly with rotational speed, while internal friction increases with the square of the speed. This partially compensates these 
two effects, and therefore satisfactory efficiency is achieved over a relatively wide range of speeds, unlike turbines where the slightest deviation from the nominal speed means a significant decrease in efficiency. If the operating point corresponds to a design point, screw expanders cannot in most cases compete with turbine efficiency. However, more stable performance over a wider operating range makes screw expanders more flexible and more advantageous than turbines in applications where operation over a wider range of conditions is required [1].

The disadvantage of screw expanders is their built-in volume ratio $\left(V_{I}\right)$. This ratio is defined by the geometry of the expander casing. The volume ratio is defined as the ratio of gas volumes in the low pressure port $\left(V_{L P}\right)$ and the high pressure port $\left(V_{H P}\right)$ [4]:

$$
V_{I}=\frac{V_{L P}}{V_{H P}}
$$

From the adiabatic equation, it can be deduced that the built-in volume ratio also corresponds to the pressure ratio:

$$
\begin{aligned}
p \cdot V^{k} & =\text { konst } \\
p_{H P} \cdot\left(V_{H P}\right)^{k} & =p_{L P} \cdot\left(V_{L P}\right)^{k} \\
\frac{p_{H P}}{p_{L P}} & =\left(\frac{V_{L P}}{V_{H P}}\right)^{k} \\
\frac{p_{H P}}{p_{L P}} & =V_{I}^{k} \\
P_{I} & =V_{I}^{k}
\end{aligned}
$$

Where $\mathrm{P}_{\mathrm{I}}$ indicates the pressure ratio and represents the discharge pressure divided by the suction pressure, i.e. the admission pressure divided by the emission pressure. The adiabatic exponent $k$ is dependent on pressure and temperature and can be determined as the ratio of specific heat defined at constant pressure and volume $\left(k=c_{p} / c_{V}\right)$. It is believed that any operation other than that corresponding to the built-in pressure ratio causes a decrease in the isentropic efficiency. At lower pressure ratios, 'over-expansion losses' occur and at higher pressure ratios, 'under-expansion losses' occur [5].

\subsection{Experimental Approach}

For characterization, the screw expander (ATMOS B100) was operated with air and integrated into a test rig. This rig consists of an oil supply system with high performance oil (Mobil Rarus 424), a generator and a compressor unit. Pressure sensors (Kistler 6061B, Kistler 6125C), temperature sensors (Pt100) and an angle encoder (AVL 365X) are used to perform the experiments necessary for the characterization. Five pressure sensors are positioned inside the screw expander to monitor the complete progress of the pressure. Additionally, one pressure sensor is mounted in the intake and one in the exhaust air duct. The temperature sensors measure the intake and exhaust air temperature.
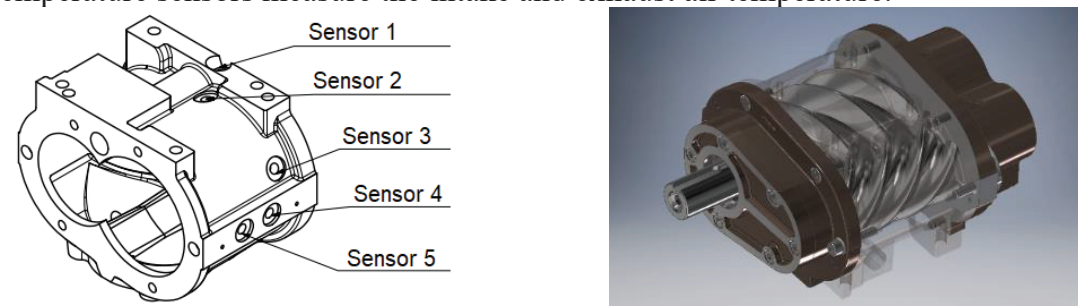

Fig. 3. Casing with drilled holes for pressure sensors (left), 3D CAD model of test bench (right). 
The angle encoder is necessary to acquire the corresponding time. In addition, a torque measuring shaft (FR-A701KTR Dataflex 32/30) was positioned between the screw expander and the generator.

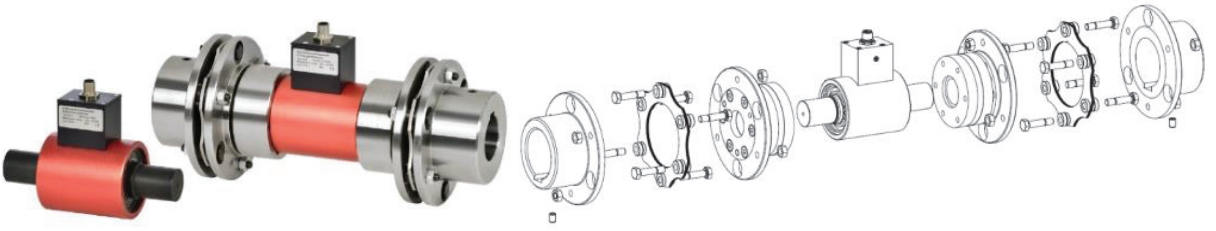

Fig. 4. Torque sensor FR-A701KTR Dataflex 32/30 [6].

During the measurements the oil temperature was $25^{\circ} \mathrm{C}$ with a corresponding flow rate of 1 1/h. The air inlet temperature was adjusted to $22^{\circ} \mathrm{C}$. In Figure 5 the pressure development over time is displayed for all four cases. It can be seen that a significant pressure drop inside the screw expander is measured within the first $60^{\circ}$ for all four cases. Then the pressure is nearly constant. More specific test conditions and the corresponding measurements are shown in Table 1. The inlet pressure and rotation speed was varied for four different test cases. All the parameters are summarized in Table 1.

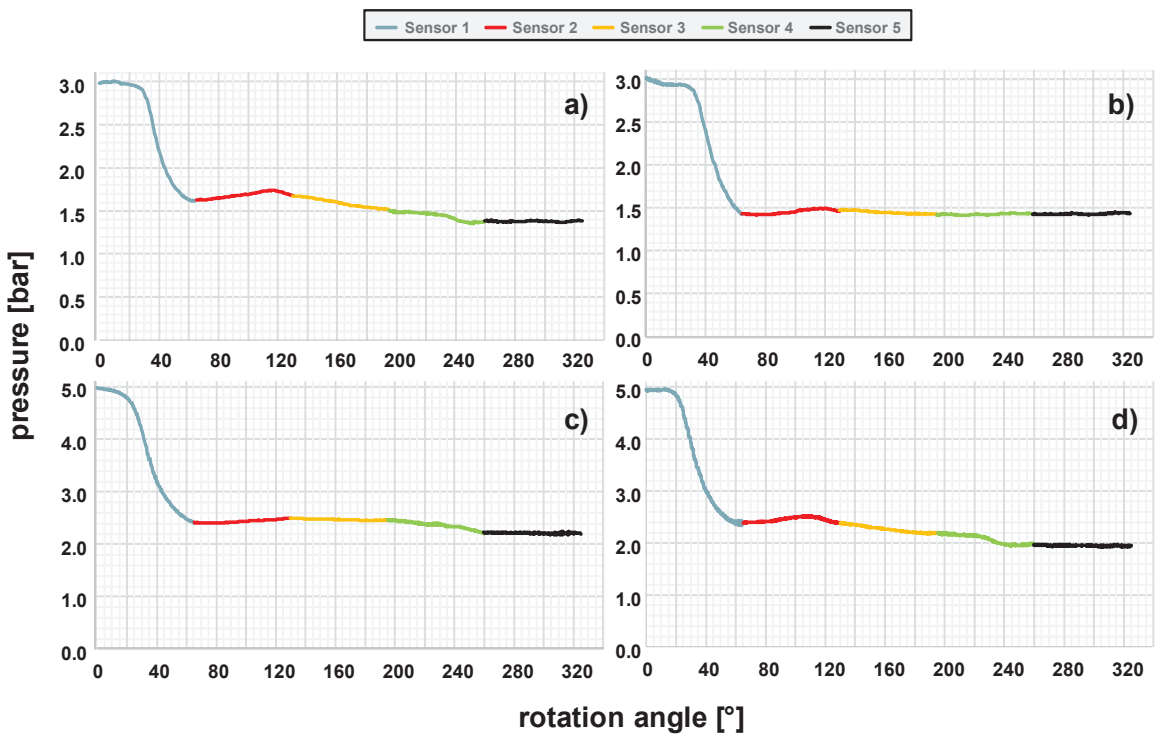

Fig. 5. Pressure development inside the screw expander.

Table 1. Parameters of the different operation points.

\begin{tabular}{|c|c|c|c|c|c|}
\hline $\begin{array}{c}\text { Measurement } \\
\text { point }\end{array}$ & $\begin{array}{c}\text { Rotation } \\
\text { speed }\end{array}$ & $\begin{array}{c}\text { Inlet } \\
\text { pressure }\end{array}$ & $\begin{array}{c}\text { Outlet } \\
\text { temperature }\end{array}$ & $\begin{array}{c}\text { Volume } \\
\text { flow }\end{array}$ & $\begin{array}{c}\text { Mechanical } \\
\text { shaft power }\end{array}$ \\
\hline & $1 / \mathrm{min}$ & bar & ${ }^{\circ} \mathrm{C}$ & $\mathrm{m}^{3} / \mathrm{h}$ & $\mathrm{W}$ \\
\hline a) & 1000 & 3 & 3 & 72 & 558 \\
\hline b) & 1500 & 3 & 3 & 83 & 609 \\
\hline c) & 1000 & 5 & -10 & 117 & 1563 \\
\hline d) & 1500 & 5 & -16 & 147 & 1987 \\
\hline
\end{tabular}


In Figure 6a the torque of the screw expander in relation to the expander speed is displayed for an inlet pressure between 2-10 bar. Negative values indicate that energy is delivered by the screw expander. As expected, the torque rises with increasing inlet pressure. For a constant inlet pressure, the torque descends linearly due to the friction. The shaft power is shown in Figure 6b. At an inlet pressure of 2 bar nearly no shaft power is measurable. At higher inlet pressure the shaft power increases with rising expander speed. At an inlet pressure of 10 bar the compressor could supply the required amount of air only up to an expander speed of $1500 \mathrm{rpm}$ and short measurement periods.

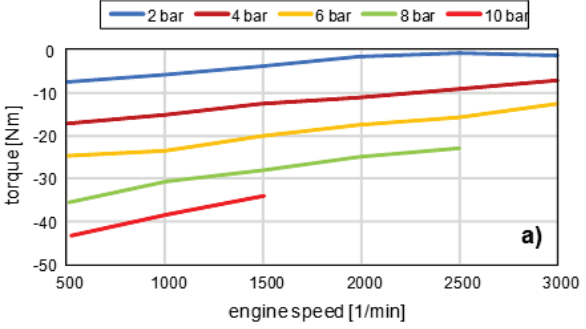

a)

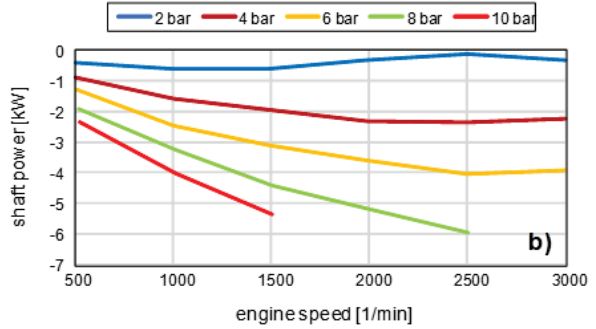

b)

Fig. 6. Development of torque (a), shaft power (b) and efficiency over engine speed.

\subsection{Mathematical model of expansion process}

The description of the working chamber shape is determined by the teeth shape of both rotors, called the 'male' and rotor and the 'female' rotor. In principle, if an imaginary section of both rotors perpendicular to the axis of rotation is made, the shapes of the rotor profiles are obtained. A precise analytical description of the female rotor profile is crucial for computer modelling the geometry. This profile can be understood as a periodic geometry that is formed by elements of the same shape (teeth) rotated by an angle of $2 \pi / \mathrm{n}$, where $n$ is the number of teeth of the female rotor. Each tooth is described by a series of consecutive analytical curves which are most often lines and circular arcs. The tooth shape of the male rotor is derived using the envelope principle, which is mathematically given by transformation of the coordinates of the contact points of both profiles. As in the case of the female rotor, the shape of the male rotor profile is composed of a periodically repeating geometry of the tooth shape. The number of teeth of the male rotor is given by the distance of the axes of rotation of both twin rotors and by the diameters of the addendum circles that together with the teeth define the monitored working chamber area in the section, see Figure 7.

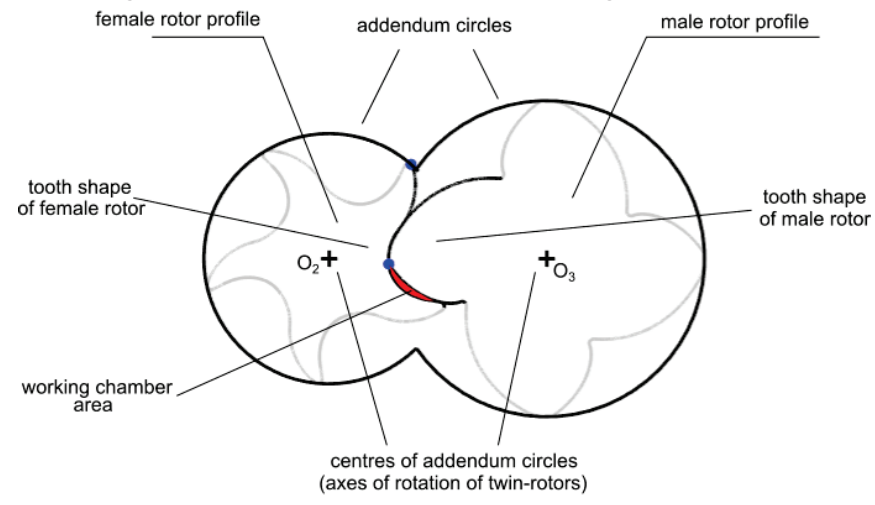

Fig. 7. Twin rotor profiles in frontal plane. 
If the twin rotors are a total length $l$, the geometry can be represented by $m$ parallel perpendicular slices. Taking a distance of two neighbouring $\Delta l_{s}, s=1, \ldots, m-1$, then the elementary volume $V_{S}$ of the working chamber is computed using

$$
V_{s}=\frac{A_{i}+A_{i+1}}{2} \Delta l_{s}, \quad i=1, \ldots, m
$$

where $A_{i}$ and $A_{i+1}$ are the working chamber areas in slices i and i+1, respectively. Then, the total volume of the working chamber is the sum of the elementary volumes along the entire length of the rotor for a given angle of rotation.

For the specified angles of rotation, the working chamber is filled through the inlet port by air mixed with added oil for lubrication. The inlet port characteristics are determined by the geometry of the twin screw expander housing. Inlet port geometry is modelled using primitive curves and its position is derived with respect to the position of the twin rotors axes of rotation. The amount of supplied working medium is thus influenced not only by the geometrical characteristics of the working chamber and the angle of rotation of the rotors, but also by the size of the cross-section area of the inlet port through which the working medium can enter the working chamber. In Figure 8, the hatched area through which the working medium enters the working chamber is marked for a chosen angle of rotation.

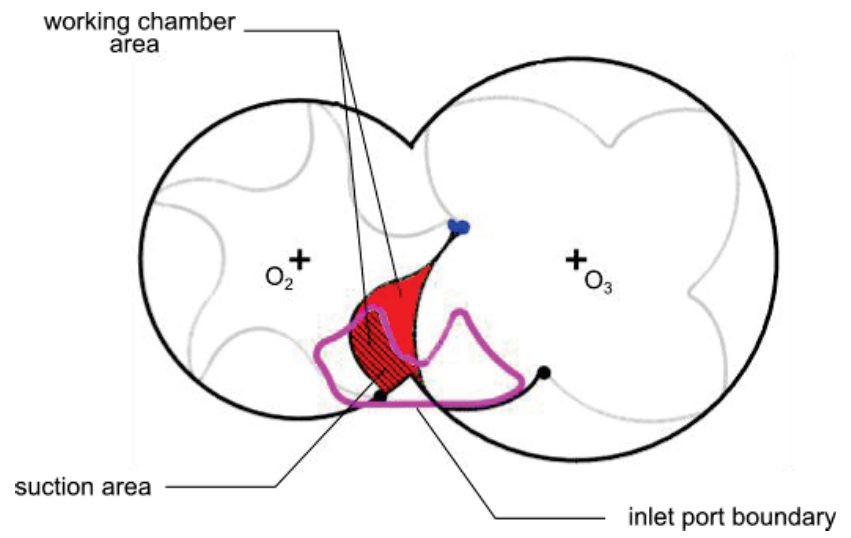

Fig. 8. Active inlet port area for a chosen angle of rotation.

The benefit of an analytical description of the geometry of the screw expander working chambers lies in its use for possible modifications to the working chamber geometry. Possible impacts of geometry changes on the expander efficiency can be evaluated, which require calculations of the expansion phase thermodynamics and especially the solution of the pressure in the working space during the expander working cycle. We developed our own simplified, computationally fast solver of the expansion phase to handle this task effectively. Our solver is based on an expansion model in an isolated single working space of the screw expander, which is derivative of the adiabatic law with the assumption of a working medium consisting of two separate phases, namely compressible gas (air) and incompressible liquid (oil). In the pure expansion phase (between injection and discharging) it can be written as

$$
p(V)=p^{I N}\left(\frac{V^{I N}-V_{\text {oil }}^{I N}}{V-V_{\text {oil }}^{I N}}\right)^{k}
$$

where $V$ is the volume of the working space changing during the expansion, $p$ is the corresponding pressure, $p^{I N}$ is the inlet pressure, $V^{I N}$ is the volume of the working space at 
the moment of closing the inlet port, $V_{\text {oil }}^{I N}$ is the total volume of the injected incompressible oil to the working space and $k$ is the heat capacity ratio. Detailed studies of the associated phenomena affecting the pressure in the working space (propagation of expansion waves, losses due to friction, centrifugal forces, etc.) have shown that when using air as the main working medium at the considered working pressures and temperatures (i.e. always using superheated air vapour), the adiabatic law expansion model is a sufficiently accurate approach for calculating the pressure inside the expanding working space. During the expansion, this model assumes the same pressure along the working space, which decreases over time as the working space increases its volume. This model of working medium expansion is a good approach when assuming fast processes (adiabatic expansion of gas without heat exchanges) and an ideal geometry with ideal contacts (no leakage flow losses). The proposed model based on the adiabatic law for the solution of the expansion phase is then incorporated in the solver in the form of a simple explicit algebraic equation.

Initial measurements of the expansion pressures on a test stand prototype working with a real screw expander clearly pointed out strong leakage flows. Likewise, outlet temperatures were not in accordance with the adiabatic expansion model predictions, which undoubtedly indicates a strong re-heating of the air during expansion. The solution provided by the current idealized model deviates significantly from the measured data when employing a real expander. To adjust the solutions of our solver to the measurement data, the idealized model was modified to capture these two observed phenomena.

The new solver works with all the active working spaces inside the screw expander simultaneously. The expander working cycle is discretized in time explicitly and the expansions in individual working spaces are calculated separately by the adiabatic expansion model between two consecutive time levels. Subsequently, the air leakage mass flow $Q_{m}^{A I R}$ between pairs of adjacent working spaces is evaluated using the proposed flow function, which assumes only air flow through the gap between the stator and both rotors, not oil leakages

$$
Q_{m}^{A I R}=f(\Delta p, h) l \Delta t,
$$

where $\Delta p$ is the pressure difference between adjacent working spaces, $h$ and $l$ are the thickness and length of the gap and $\Delta t$ the time step size, the flow function is continuous and smooth and satisfies conditions $f(0, h) \equiv 0$ and $f(\Delta p, 0) \equiv 0$. The leakage flow function is a result of $1402 \mathrm{D}$ simulations in Fluent for various $\Delta p$ and $h$, starting from the main rotor geometry. The proposed leakage flow sub-model assumes equal leakages through the male and female rotor-stator gaps.

Furthermore, the heat transfer between the air and oil takes into account getting the air outlet temperature into accordance with the measurement data from the test stand. However, we still assume a fully heated expander, and therefore the heat exchange with the stator or the environment is not considered. The air and oil temperatures are thus adjusted in individual time levels according to the relations for interphase heat exchange given in [7] and the corresponding air pressure is corrected.

The expansion and the heat transfer are thus solved in all the working spaces individually, but all the active working spaces are interconnected in pairs through gaps where air leakage flow may occur. A similar solver is commonly used in some computational software e.g. [8]. The solution algorithm handles all the active working spaces together, which can generally occur in different phases of the work cycle: forming the working space, filling the chamber through the inlet port, pure expansion, forming a blow-hole and direct opening of the outlet port. During these parts of the working cycle, the volume of the working space is increasing. The thermodynamic state is calculated from the formation of the working chamber, before the inlet opening. After that, the amount of air through the inlet port with time-changing cross-section area is reduced on the basis of the theory of compressible flow in nozzles. 
Finally, the calculations end with the opening of the blow-hole, when the expanding working space starts to be connected to the outlet port via the blow-hole and the older depressurized working space. We assume that the outlet pressure does not decrease further. This assumption is in good agreement with the measured data.

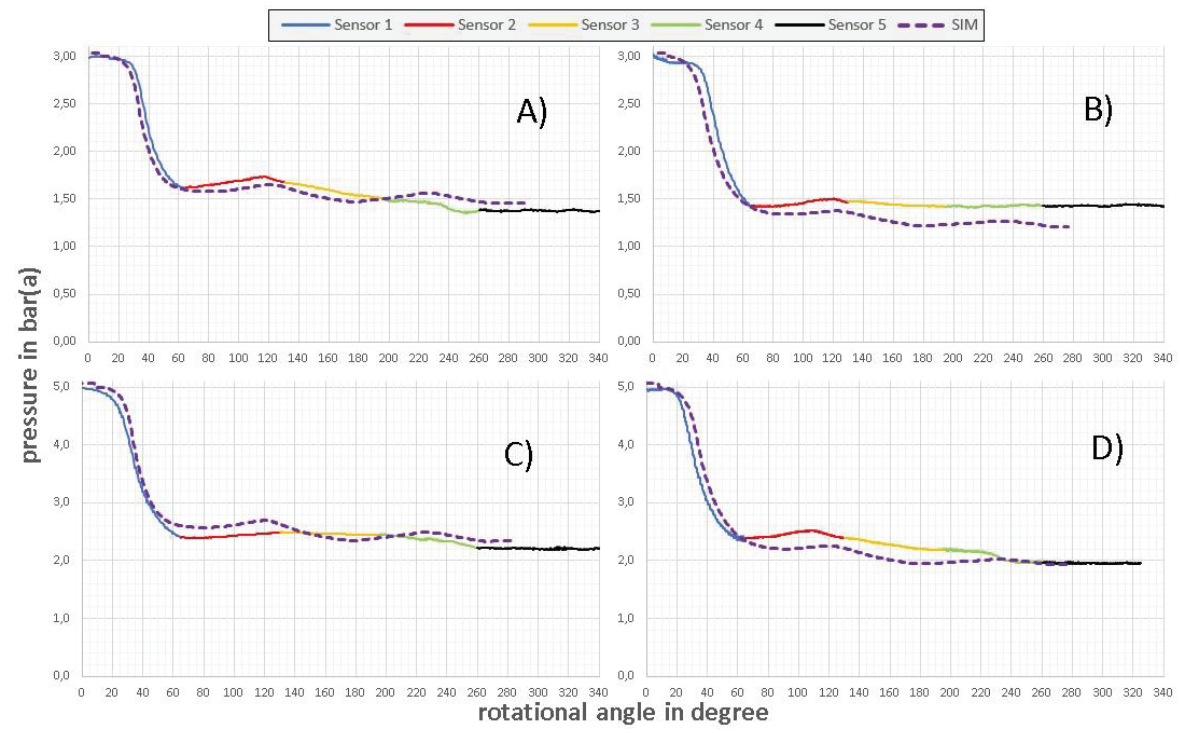

Fig. 9. Comparison of the measured pressures and the solutions of numerical simulation (dashed purple line).

Table 2. Outlet temperature and standardized volume flow rate of air calculated from numerical simulations at different operation points.

\begin{tabular}{|c|c|c|c|c|}
\hline $\begin{array}{c}\text { Measurement } \\
\text { point }\end{array}$ & $\begin{array}{c}\text { Rotation } \\
\text { speed }\end{array}$ & $\begin{array}{c}\text { Inlet } \\
\text { pressure }\end{array}$ & $\begin{array}{c}\text { Outlet } \\
\text { temperature }\end{array}$ & $\begin{array}{c}\text { Volume } \\
\text { flow }\end{array}$ \\
\hline & $1 / \mathrm{min}$ & bar & ${ }^{\circ} \mathrm{C}$ & $\mathrm{m}^{3} / \mathrm{h}$ \\
\hline A) & 1000 & 3 & -11 & 70 \\
\hline B) & 1500 & 3 & -12 & 85 \\
\hline C) & 1000 & 5 & -25 & 117 \\
\hline D) & 1500 & 5 & -26 & 143 \\
\hline
\end{tabular}

\subsection{CFD Simulations of screw expander}

CFD simulations should be conducted to obtain detailed information about fluid flow and the expansion process between the screw rotors. Furthermore, in the later stages, these simulations should also include oil lubrication and thus they should provide information regarding oil injection, its distribution between the rotors as well as the heat transfer between the oil and the expanded gas. As of now, simpler CFD simulations have been conducted as a proof-of-concept that the intended simulations are feasible with the computational resources and simulation software we have available (we will use ANSYS Fluent since we do not possess any single-purpose simulation software for screw machines). 
Below we will briefly discuss the geometry of the screw expander which was obtained by $3 \mathrm{D}$ scanning the real device. Then we will discuss the approach for 3D simulations and the analyses will be presented.

\subsubsection{D Scanning of Screw Expander}

The basis of each CFD simulation is the geometry (aka computational domain) which, in our case, was obtained by 3D scanning a real screw expander. A real device was disassembled, and each component was scanned using precise industrial 3D metrology, ATOS Core. It uses a stereo camera set-up working on the principle of triangulation. The result of the scanning can be seen in Fig 10. The inverse geometry of these solid components (i.e., the volume between the rotors inside the housing) is the computational domain for subsequent CFD simulations.

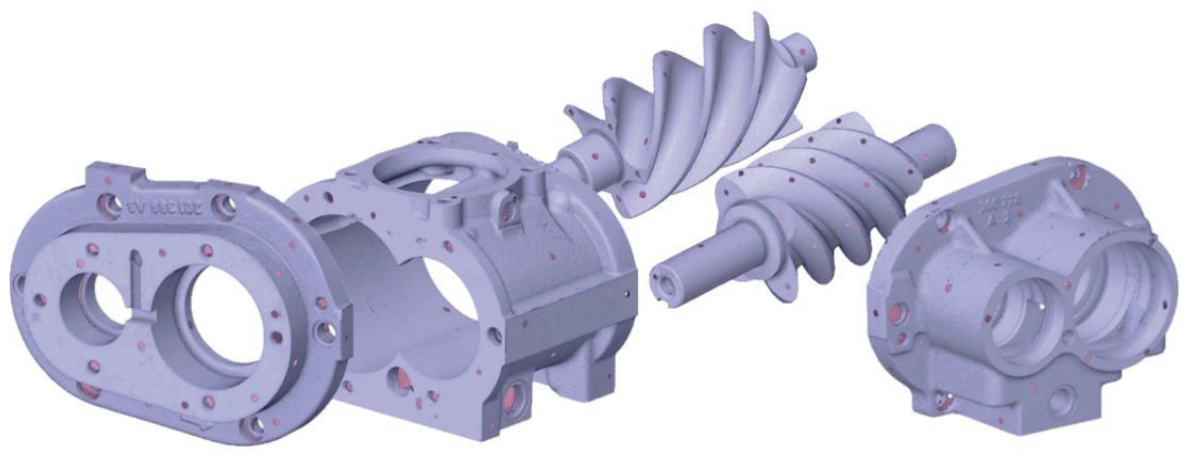

Fig. 10. 3D scan of screw expander.

\subsubsection{Overview of CFD Analyses}

CFD simulations of the screw expander in ANSYS Fluent can be done either using dynamic mesh or overset mesh. The former means that after each slight rotation (the size will depend on the allowed deformation of the mesh elements), the computational domain will need to be re-meshed. It follows that this procedure significantly increases the requirements on the computational resources and the overall simulation time. The latter approach does not require re-meshing as the simulation model consists of two meshes that move onto each other. However, it requires very dense meshes so that the elements overlay each other, but this approach seems to be very reliable for 3D simulations based on our previous experience.

For our simulations, we decided to first use the dynamic mesh. The main challenge in the dynamic mesh simulation lies in the proper setup of the element size and their allowed deformation before the re-meshing process. It goes without saying that several simulations must be done to verify the setup and thus a simpler domain should be utilized to decrease the computational demands. We decided to start with a $2 \mathrm{D}$ simulation to get the sizing of the elements and the values of the allowed deformation. Then we applied the settings to a 3D segment (created by cutting the volume inside the housing and between the rotors by two parallel planes perpendicular to the axes of rotation of the rotors) thus verifying the settings in 3D. Finally, the entire domain is simulated. This concept is shown in Fig. 11. 


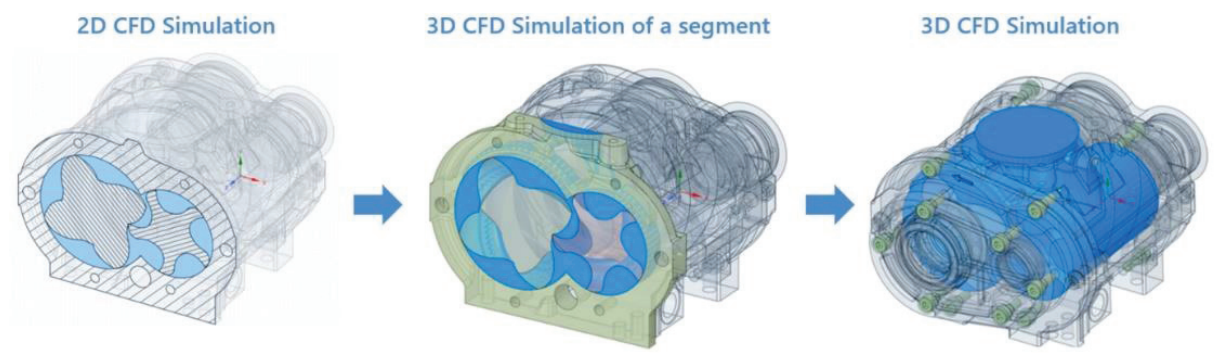

Fig. 11. Overview of CFD simulations

\subsection{3 $2 D$ and $3 D$ CFD analyses}

As of now, 2D and 3D simulations of a segment have been performed. Both simulations were done with a time-step of $0.002 \mathrm{~ms}$ (i.e., it requires 10000 time-steps per 1 rotor revolution) and a two-equation SST k- $\omega$ turbulence model was used. The mesh is shown in Figure 12a and some missing areas can be seen in locations where the teeth are close to the housing wall, or they are close to each other. This is on purpose, as we do not intend to model the leakage in these initial simulations. Gap detection was used to remove these small gaps from the simulation.

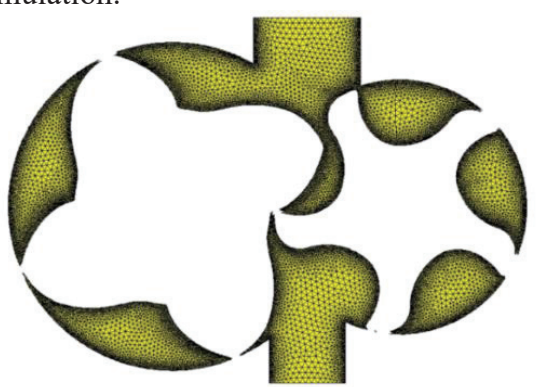

a)

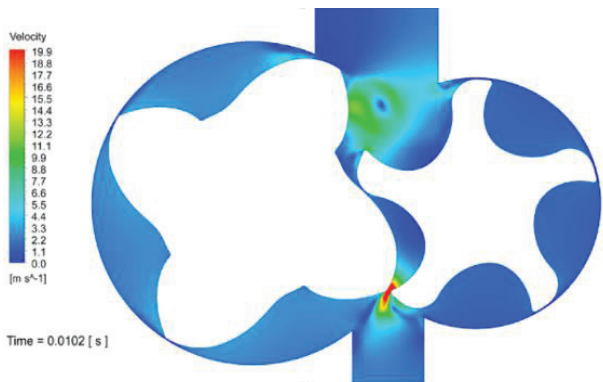

b)

Fig. 12. a) Computational mesh for $2 \mathrm{D}$ simulation. b) Results of 2D CFD simulation (velocity field).

In Figure $12 b$ the velocity field can be seen for a specific time step. Further analysis of the results will not be presented in this section as it would be outside the scope of this article. To conclude, it should be noted that the mesh settings from the $2 \mathrm{D}$ analysis were applied to the $3 \mathrm{D}$ analysis of the segment and the simulation was stable and converged very well. However, the simulation time was not acceptable (a few days per rotor revolution), and thus further optimization is necessary before the simulation of the entire domain.

CFD simulations become much more complex when oil flow is considered. Multiphase flow requires a further increase in computing capacity. The most common approaches for solving multiphase flow using CFD solvers are the Euler-Euler (gas and liquid are interpenetrating continuous media) and Euler-Lagrangian (gas is a continuous medium and liquid is dispersed phase) approaches [9].

\section{Conclusion}

The joint implementation of the project by the University of West Bohemia, OTH AmbergWeiden and ATMOS resulted in an initial analysis of the expansion process inside an oilflooded air screw expander. Follow-up activities will use the established Design of Experiment and the developed mathematical model for further analysis of the screw expander 
in real conditions of the power unit. Real conditions are characterized mainly by a special working medium in the form of low-boiling organic matter, the compatibility of which with lubricating oil and sealing elements of the expander must be taken into account. The real conditions differ from the performed analysis in operating temperatures, when temperatures around $100{ }^{\circ} \mathrm{C}$ are reached at the inlet to the expander. In order to achieve satisfactory performance in real conditions, the speed of the expander must be higher, which increases the medium flow. At higher media flows, problems can occur in separating the oil from the working fluid. The next step in terms of the numerical approach will be the development of a 3D model, which is very demanding given the need to use a dynamic computing mesh.

The presented work was financially supported by the European Commission and the European Regional Development Fund. The aim of the project is to strengthen cross-border activities and cooperation in the field of research and innovation between Czech Republic and Free State of Bavaria.

\section{References}

1. Smith, I., Stosic, N., Kovacevic, A. Power recovery from low-grade heat by means of screw expanders. Cambridge, UK: Woodhead Publishing, [2014]. Woodhead Publishing in mechanical engineering. ISBN 978-1-78242-189-4.

2. Iva Papes, Joris Degroote, Jan Vierendeels, New insights in twin screw expander performance for small scale ORC systems from 3D CFD analysis, Applied Thermal Engineering, Volume 91, 2015, Pages 535-546, ISSN 1359-4311, https://doi.org/10.1016/j.applthermaleng.2015.08.034.

3. Olivier Dumont, Rémi Dickes, Vincent Lemort, Experimental investigation of four volumetric expanders, Energy Procedia, Volume 129, 2017, Pages 859-866, ISSN 18766102, https://doi.org/10.1016/j.egypro.2017.09.206.

4. Wennemar Jurgen (2009). Dry Screw Compressor Performance And Application Range. Texas A\&M University. Turbomachinery Laboratories. Available electronically from http : / /hdl .handle .net /1969.1/163089.

5. Hsu, Sung-Wei \& Chiang, Hsiao-Wei \& Yen, Chih-Wei. (2014). Experimental Investigation of the Performance of a Hermetic Screw-Expander Organic Rankine Cycle. Energies. 7. 6172-6185. 10.3390/en7096172.

6. KTR-Group, DATAFLEX. Drehmomentmesswelle der Bauart 32/300, handbuch.

7. Brennen, C. E., Fundamentals of Multiphase Flows, Cambridge University Press, 2005. ISBN 0521848040.

8. Stosic, N., Smith, I and Kovacevic, A. (2005) Screw Compressors - Mathematical Modelling and Performance Calculation. Springer Berlin Heidelberg New York. ISBN10 3-540-24275-9.

9. Basha, Nausheen \& Rane, Sham \& Kovacevic, Ahmed. (2018). Multiphase Flow Analysis in an Oil-injected Twin Screw Compressor. Proceedings of the 3rd World Congress on Momentum, Heat and Mass Transfer (MHMT'18). DOI: 10.11159/icmfht18.132. 